\title{
Accuracy of retrieving temperature and humidity profiles by ground-based microwave radiometry in truly complex terrain
}

\author{
G. Massaro ${ }^{1}$, I. Stiperski ${ }^{2}$, B. Pospichal ${ }^{3}$, and M. W. Rotach ${ }^{2}$ \\ ${ }^{1}$ Twist-Off Srl, Padua, Italy \\ ${ }^{2}$ Institute of Meteorology and Geophysics, University of Innsbruck, Austria \\ ${ }^{3}$ Institute for Meteorology, University of Leipzig, Germany \\ Correspondence to: G. Massaro (giovanni.massaro@teletu.it)
}

Received: 3 February 2015 - Published in Atmos. Meas. Tech. Discuss.: 2 March 2015

Revised: 18 July 2015 - Accepted: 3 August 2015 - Published: 19 August 2015

\begin{abstract}
Within the Innsbruck Box project, a groundbased microwave radiometer (RPG-HATPRO) was operated in the Inn Valley (Austria), in very complex terrain, between September 2012 and May 2013 to obtain temperature and humidity vertical profiles of the full troposphere with a specific focus on the valley boundary layer. In order to assess its performance in a deep alpine valley, the profiles obtained by the radiometer with different retrieval algorithms based on different climatologies are compared to local radiosonde data. A retrieval that is improved with respect to the one provided by the manufacturer, based on better resolved data, shows a significantly smaller root mean square error (RMSE), both for the temperature and humidity profiles. The improvement is particularly substantial at the heights close to the mountaintop level and in the upper troposphere. Lower-level inversions, common in an alpine valley, are resolved to a satisfactory degree. On the other hand, upper-level inversions (above $1200 \mathrm{~m}$ ) still pose a significant challenge for retrieval. For this purpose, specialized retrieval algorithms were developed by classifying the radiosonde climatologies into specialized categories according to different criteria (seasons, daytime, nighttime) and using additional regressors (e.g., measurements from mountain stations). The training and testing on the radiosonde data for these specialized categories suggests that a classification of profiles that reproduces meaningful physical characteristics can yield improved targeted specialized retrievals. A novel and very promising method of improving the profile retrieval in a mountainous region is adding further information in the retrieval, such as the surface temperature at fixed levels along a topographic slope or from nearby mountaintops.
\end{abstract}

\section{Introduction}

Precise knowledge of the temperature and humidity structure of the atmosphere is essential not only for numerical weather prediction but also for the study of small-scale phenomena within the atmospheric boundary layer. Especially in complex terrain the boundary layers are still a topic of intense research, and the information about the vertical profiles, such as temperature and humidity, as well as their temporal evolution is particularly valuable (e.g., Rotach and Zardi, 2007). For this latter topic the temporal scales of minutes and spatial scales on the order of few tens to hundreds of meters are required.

One of the most accurate methods for measuring atmospheric vertical profiles of temperature and humidity are radio soundings, but given their cost as well as finite space/time resolution (routinely once or twice per day), they are inadequate for a detailed study of the diurnal evolution of the nearsurface atmosphere. An alternative to obtaining such profiles is the use of remote sensing. Passive remote sensing from satellites, exploiting infrared or microwave radiation naturally emitted by atmospheric constituents, has the advantage of covering areas with sparse data (e.g., oceans). The vertical resolution is fairly good in the upper troposphere; however the information deteriorates towards the ground, due to the satellite's viewing geometry and the opacity of thick clouds (Zhou et al., 2007); in addition, the surface contribution to the signal has to be disentangled from the atmospheric signal, which is more difficult close to the ground. For example, Divakarla et al. (2006) compared temperature profiles from an atmospheric infrared sounder (AIRS) with global radiosonde 
data for 2 years, finding a root mean square error (RMSE) larger than $1.5 \mathrm{~K}$ at ground level. Thus, the accuracy of passive remote sensing from satellites is too poor to be useful for studying boundary layer evolution.

On the other hand, ground-based remote sensing can provide valuable information on the temperature and humidity structure of the lower troposphere. Methods using passive microwave radiometry have rapidly developed within the last decade when radiometers became commercially available. The measurement principle of a ground-based passive radiometer is measuring the radiation emitted by the atmospheric constituents (oxygen, water vapor, cloud droplets). Unlike infrared spectrometers that are able to produce vertical profiles only under clear-sky conditions due to strong liquid water absorption, a microwave spectrometer operates under almost all conditions (Löhnert et al., 2009) and is therefore more appropriate for long-term monitoring of a valley atmosphere. One of commercially available radiometers is the RPG-HATPRO (Humidity And Temperature PROfiler) manufactured by Radiometer Physics GmbH (Rose et al., 2005). Statistical algorithms are used to retrieve humidity and temperature vertical profiles from the observed brightness temperatures. These retrieval algorithms exploit a local history of pressure-temperature-humidity profiles by using a multiple regression approach following Löhnert and Crewell (2003) and Crewell and Löhnert (2007). Other training data have been proposed. For example, Güldner (2013) introduced a new approach for the retrieval development, namely using data from the COSMO numerical weather prediction model (Steppeler et al., 2003). He showed that with this approach retrieved profiles almost reach the quality of those based on a radiosonde training data set. An additional advantage of such an approach is that data for retrieval are available "always and everywhere" (at each grid point of an operational model). However, using model data introduces additional uncertainty especially in complex terrain due to grid resolution and steep(er) slopes as well as finite forecasting skill.

The advantages of profiles derived from passive microwave observations over radiosonde data, beyond the economic convenience, are their high temporal resolution (on the order of $1 \mathrm{~min}$ or less), their vertical resolution comparable to current operational numerical weather prediction models and their ability to operate under all weather conditions, because clouds are semi-transparent for the microwave frequency range, with the exception of precipitation (Löhnert et al., 2009). Crewell and Löhnert (2007) compared RPG-HATPRO observations at Lindenberg (Germany) with data from 80 nearby radiosondes over flat terrain. Löhnert and Maier (2012) did a 3.5-year statistical comparison between 2107 radio soundings and RPG-HATPRO temperature profiles at the lowland station of Payerne (Switzerland). They found accuracies between 0.4 and $0.8 \mathrm{~K}$ in the lowest $500 \mathrm{~m}$, gradually deteriorating with height $(1.2 \mathrm{~K}$ at $1200 \mathrm{~m}$ and $1.7 \mathrm{~K}$ at $4000 \mathrm{~m}$ above ground).
In complex terrain, Sanchez et al. (2013) operated a MP3000A microwave radiometer in Sierra Guadarrama (Spain). Comparison with radiosondes launched at a distance of $50 \mathrm{~km}$ provided accuracies of about $2 \mathrm{~K}$ in the lower troposphere. A MTP-5HE (by ATTEX) was used in the Bergen Valley (Wolf et al., 2014) in order to obtain temperature profiles and compare them with 1-year measurements from two automatic meteorological stations, located at the ground and on a mountain about $550 \mathrm{~m}$ above the valley floor; they obtained an RMSE of 0.2 and $0.8 \mathrm{~K}$ for the two stations, respectively. Overall, a thorough assessment of a passive temperature profiler in complex terrain using close-by radiosondes seems to be missing. Furthermore, operation of a passive microwave profiler in a deep valley raises the question as to what the potential impact of slopes in the immediate surrounding of the profiler might be. While the atmospheric temperature profile is variable in time (what is, of course, the target of the observations) the ground has usually a larger thermal inertia than air, thus potentially degrading the statistical retrieval algorithm of the profiler.

Regarding the performance of retrieving absolute humidity profiles, Löhnert et al. (2009) compared the absolute humidity profiles obtained from simulated measurements by such a radiometer with radiosonde data at the aerological station of Payerne. They found an RMSE on the order of $0.8 \mathrm{~g} \mathrm{~m}^{-3}$ in the lowest $500 \mathrm{~m}$, around $0.7 \mathrm{~g} \mathrm{~m}^{-3}$ at $1200 \mathrm{~m}$ and about $0.4 \mathrm{~g} \mathrm{~m}^{-3}$ at $4000 \mathrm{~m}$ above ground. Sanchez et al. (2013) found comparable accuracies for more complex terrain.

Within the Innsbruck Box project (Rotach et al., 2015), a RPG-HATPRO has been operated since 2012 in Innsbruck (Austria), located in the Inn Valley, an approximately eastwest-oriented $1700 \mathrm{~m}$ deep U-shaped alpine valley (Fig. 1). As far as the authors know, this type of a radiometer has never been used for a prolonged period of time in such a truly complex setting where inversions (near surface and especially near mountaintop) pose a significant challenge to the radiometer measurements and where regular radio soundings are available. The questions that we specifically want to address are whether a radiometer can provide useful profiles in complex terrain and how accurate the retrieved profiles are in comparison to radiosonde data. In particular, we are interested in the performance of the radiometer within the valley boundary layer. The employment of ground-based radiometry in an alpine valley is especially challenging because the topography strongly affects the boundary layer dynamics and temperature structure though formation of strong inversions, differential solar heating (Matzinger et al., 2003), anabatic/katabatic winds (Zardi and Whiteman, 2012), secondary cross-valley circulation (Weigel and Rotach, 2004), etc. Given that microwave radiation may originate not only from "the sky" but also from the surrounding mountains and that all retrieval methods assume horizontal homogeneity of the atmosphere, which is not satisfied in an alpine valley, it is necessary to assess the performance and accuracy of the 
ground-based radiometer in complex terrain. Unfortunately, scans in different azimuth directions needed for obtaining a 3-D-temperature field are not possible in the present environment, since the instrument would soon have the nearby mountains in its direct optical path if it pointed under lower elevation angles towards north or south. With this in mind we wish to examine the possibility of improving the retrieval algorithm by including additional regressors, such as temperature observations along the slopes or on mountaintops.

Section 2 describes the basics of microwave radiometry, radiative transfer, as well as the retrieval algorithms. In Sect. 3 the experimental setup is explained. The comparison between the profiles obtained from the microwave radiometer by applying different retrieval algorithms with radiosonde data for 84 days between September 2012 and May 2013 is presented in Sect. 4. A final discussion and open challenges are given in Sect. 5.

\section{Microwave radiometry and retrieval development}

\subsection{Radiative transfer}

In the microwave region of the electromagnetic spectrum (about 3-300 GHz, $1 \mathrm{~mm}$ to $10 \mathrm{~cm}$ wavelength), a groundbased receiver measures the thermal radiation emitted by the atmospheric constituents, especially $\mathrm{O}_{2}$ and $\mathrm{H}_{2} \mathrm{O}$ (the latter in both gaseous and liquid form), and the cosmic background radiation. With the assumptions of a plane-parallel atmosphere, thermodynamic equilibrium and negligible scattering (valid for frequencies below $90 \mathrm{GHz}$ in the absence of rain) the brightness temperature $T_{\mathrm{b}}$ at the ground for each frequency $v$ is given by

$T_{\mathrm{b}}(v)=T_{b 0} e^{-\tau\left(s_{\mathrm{t}}\right)}+\int_{0}^{s_{\mathrm{t}}} \alpha(s) T(s) e^{-\tau(s)} \mathrm{d} s$,

with $s$ being the path length, $\alpha$ the absorption coefficient and $\tau$ the optical depth. The first term in Eq. (1) originates from the cosmic background radiation attenuated over the path of propagation. The second term represents the integral from the top of the atmosphere to the ground of the emission by atmospheric gases and hydrometeors in a particular atmospheric layer that is attenuated by the underlying atmospheric layers (Janssen, 1993).

\subsection{Retrieval algorithms for atmospheric quantities}

In order to obtain atmospheric quantities from observed brightness temperatures, the radiative transfer equation (Eq. 1) has to be inverted. However, since this is an ill-posed problem, it cannot be solved analytically. Therefore, statistical methods have to be applied, such as multiple regression or neuronal networks. The algorithms we use for this work are based on a set of training data from historical profiles (climatology) and allow retrieval of vertical profiles of temperature and humidity (Löhnert and Crewell, 2003). In our case, this set of training data consists of profiles of pressure, temperature and relative humidity $(p-T-\mathrm{RH})$ obtained by radiosonde measurements. Under the assumption of a horizontally homogeneous atmosphere, a profile consists of several layers characterized by their proper temperature; thus the integral in Eq. (1) can be converted into a sum. Theoretical $T_{\mathrm{b}}$ from the training set of radiosonde data is computed with a non-scattering radiative transfer model using gas absorption following Rosenkranz (1998) and the liquid cloud absorption according to Stogryn et al. (1995). Liquid clouds are assumed to exist above a $95 \%$ relative humidity threshold with a temperature warmer than $243.15 \mathrm{~K}$. In this case the liquid water content is calculated following the modified adiabatic model by Karstens et al. (1994).

A linear regression between these modeled $T_{\mathrm{b}}$ and temperatures at some pre-defined atmospheric layers can then be performed. These regression coefficients and $T_{\mathrm{b}}$ measured by the radiometer are then used to retrieve the atmospheric profiles also in the absence of radio soundings. The output (Out $\left.{ }^{i}\right)$ of the retrieval algorithm, which is the temperature or the absolute humidity of the $i$ th atmospheric layer, is related to the measured $T_{\mathrm{b}}$ at the $j$ frequencies in a linear and a quadratic term, respectively, and to a number of additional regressors (such as surface pressure or temperature sensors) that are introduced to potentially improve the statistical performance. The regression equation is given by Eq. (2):

$$
\begin{aligned}
& \text { Out }^{i}=C_{\mathrm{Os}}^{i}+\sum_{\text {freq, }, j} C_{\mathrm{Tb}, 1}^{i j} \mathrm{~Tb}_{j}+\sum_{\text {freq }, j} C_{\mathrm{Tb}, 2}^{i j} \mathrm{~Tb}_{j}^{2} \\
& +\sum_{\mathrm{sens}, j} C_{\mathrm{Sr}, 1}^{i j} \mathrm{Sr}_{j}+\sum_{\mathrm{sens}, j} C_{\mathrm{Sr}, 2}^{i j} \mathrm{Sr}_{j}^{2}
\end{aligned}
$$

where $C_{\mathrm{Os}}^{i}$ is an offset, $C_{\mathrm{Tb}, 1}^{i j}$ and $C_{\mathrm{Tb}, 2}^{i j}$ are the coefficients for the linear and quadratic terms, respectively, of the brightness temperatures $\mathrm{Tb}_{j}, C_{\mathrm{Sr}, 1}^{i j}$ and $C_{\mathrm{Sr}, 2}^{i j}$ are the coefficients (linear and quadratic, respectively) for the sensors at the ground level $\mathrm{Sr}_{j}$ and the index $i$ runs over several layers of the atmosphere. The vertical grid (Table 1) for which the profiles are retrieved depends on vertical resolution of the radiometer and is gradually getting coarser with height. In our case, the elevation angles used for scanning are $90.0^{\circ}, 30.0^{\circ}, 19.2^{\circ}$, $14.4^{\circ}, 11.4^{\circ}, 8.4^{\circ}, 6.6^{\circ}, 5.4^{\circ}, 4.8^{\circ}$, and $4.2^{\circ}$.

The retrieval provided by the manufacturer that we refer to as "RPG algorithm" uses radiosonde profiles at Innsbruck airport from 1999 to 2005 and from 2009 to 2012, with a low vertical resolution (i.e., standard and significant levels only) as a training data set. The radiosonde measurements are only available during nighttime (02:00 UTC). Although this significantly limits the climatology, the more challenging nighttime near-surface atmosphere with frequent inversions is still well-represented. The algorithm also takes into account measurement errors of the $T_{\mathrm{b}}$ introducing a randomly distributed noise with a standard deviation of $0.35 \mathrm{~K}$ (i.e., noise level). 


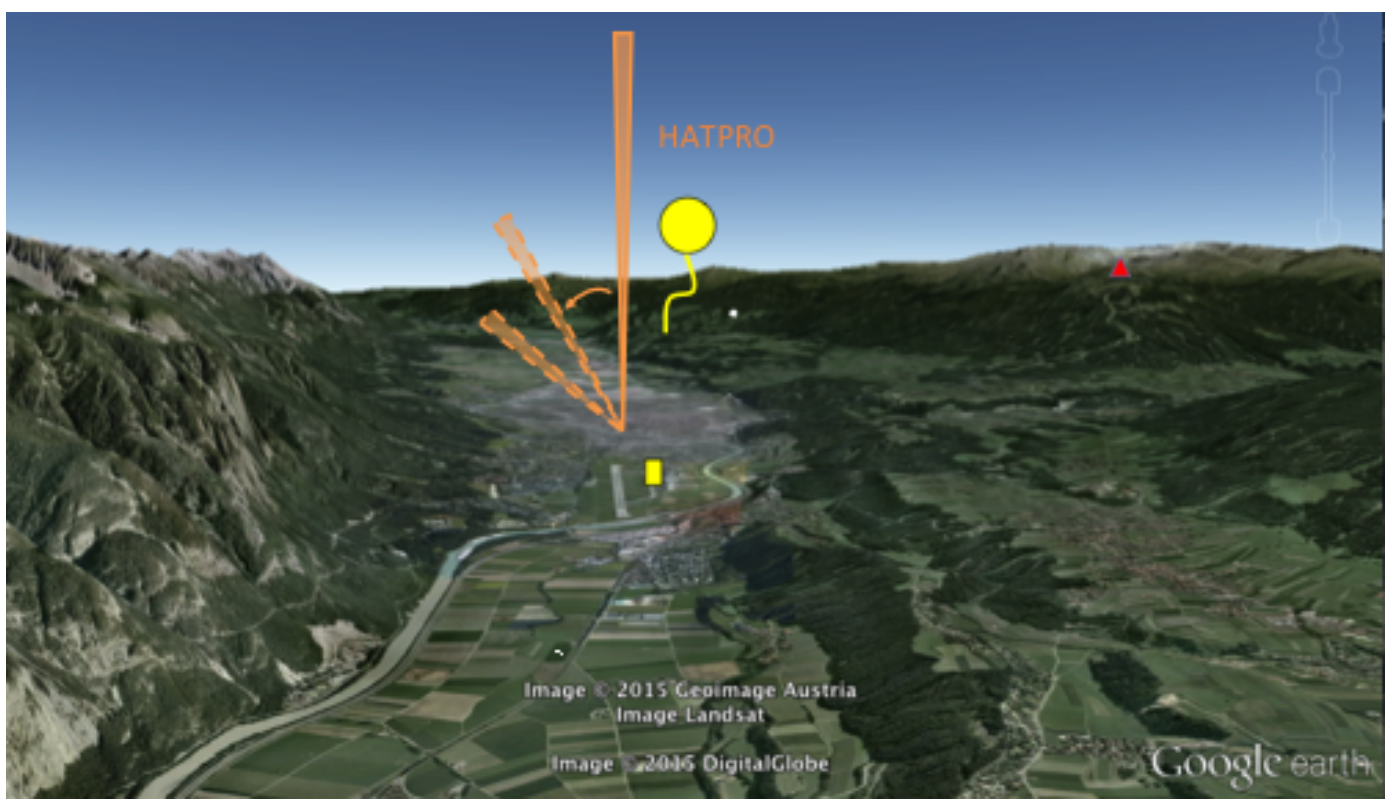

Figure 1. View from the west to the city of Innsbruck. In the foreground the airport is visible where the radio soundings are launched and the approximate position of the HATPRO on the roof of the university building with the scanning direction towards the east is indicated. The red triangle denotes the Patscherkofel mountaintop station.

Table 1. Height grids of the temperature/humidity profile retrieval, expressed in meters over the instrument.

\begin{tabular}{l}
\hline Height grids of profiles retrieved from RPG-HATPRO \\
\hline $0,10,30,50,75,100,125,150,200,250,325,400,475,550,625,700,800,900,1000,1150,1300,1450$, \\
$1600,1800,2000,2200,2500,2800,3100,3500,3900,4400,5000,5600,6200,7000,8000,9000,1000$
\end{tabular}

Only the surface pressure is used as an additional regressor. Table 2 summarizes the main characteristics of the RPG algorithm.

\subsection{Improving the retrieval algorithm}

The first step in enhancing the retrieval performance is to use a more statistically robust set of radiosonde data. The latter are now used at their full temporal resolution: $10 \mathrm{~s}$ for the soundings before 2009 and $2 \mathrm{~s}$ for the later ones, corresponding to a height resolution of $40-50$ and $8-10 \mathrm{~m}$, respectively. Additionally about 130 daytime soundings from the MAP (Bougeault et al., 2001) and ALPNAP (Harnisch et al., 2009) international projects, launched between 1999 and 2005 from the airport of Innsbruck, are used to include information on the diurnal variation. A total number of 3157 radiosonde profiles passed the quality check (cf. Sect. 3.2) and are used in the radiative transfer model. In addition, the best choice of regression (linear vs. quadratic) is tested, and the performance of the retrieval algorithm for different noise levels is investigated in order to determine the optimal value. The resulting retrieval will be denoted "IMGI algorithm" in the following (see Table 3).
The historic sounding data set is split into two subsets: the training set is used to obtain the regression coefficients, and the testing set to test the performance of the new retrieval. The subsets are chosen randomly: $70 \%$ of the total soundings for training and $30 \%$ for testing. Since several retrievals are obtained (see the following sub-section), the algorithm is considered to have an improvement over the other algorithms only if the RMSE between radiosonde and retrieved profile decreases by $0.1 \mathrm{~K}$ and more. After the validation, the best retrieval is applied to the actual radiometer data.

\subsubsection{Specialized retrieval algorithms}

The entire radiosonde data set is, naturally, very heterogeneous, spanning different seasons, times of day, atmospheric stability, etc. Löhnert and Maier (2012) tested their retrieval for different meteorological conditions such as inversions, front passages, cold or warm extremes cases and they found different results depending on the weather conditions: in particular, their retrieval showed less skill for inversion cases. However, they made no distinction in their training radiosonde data pool. Constraining the training-testing statistics to certain more homogeneous subsets (i.e., sub-sets containing profiles of similar characteristics) may be expected 
Table 2. Main characteristics of the built-in retrieval (RPG algorithm). "Ibk" stands for the operational radiosonde at the airport of Innsbruck. "Degree" refers to the order of the employed regression (see Eq. 2).

\begin{tabular}{|c|c|c|c|c|}
\hline \multirow{2}{*}{$\begin{array}{l}\text { RPG retrieval al- } \\
\text { gorithm }\end{array}$} & Training data & Degree & Surface sensor & Noise level (K) \\
\hline & $\begin{array}{l}\text { Radiosonde Ibk } \quad(\sim 3000) \\
\text { 1999-2005, 2009-2012 } \\
\text { low vertical resolution night- } \\
\text { time }\end{array}$ & 2 & pressure & 0.35 \\
\hline
\end{tabular}

Table 3. Main characteristics of the improved retrieval (IMGI algorithm) for temperature $(T)$ and absolute humidity (a). "Ibk" stands for the operational radiosonde at the airport of Innsbruck. "Degree" refers to the order of the employed regression (see Eq. 2).

\begin{tabular}{lllll}
\hline IMGI retrieval algorithm & Training data & Degree & Surface sensor & Noise level (K) \\
\cline { 2 - 5 } & Radiosonde Ibk (3157) & $T: 2$ & $T:-$ & $T: 0.20$ \\
& $\begin{array}{l}\text { 1999-2005, 2009-2012 } \\
\text { resolution 2 s/10s }\end{array}$ & $a: 2$ & $a:$ pressure & $a: 0.35$ \\
& night- and daytime & & & \\
\hline
\end{tabular}

to provide better statistics and to be beneficial to the performance of the retrieval algorithm. We will test the specialized retrievals for (1) seasons and (2) daytime and nighttime. December, January, February and March are thereby considered "winter months" and June, July, August and September "summer months". October through March are considered "cold months" and the remaining ones "warm months". All the radiosonde launches performed between sunrise and sunset are considered daytime launches and vice versa.

\section{Instrumentation}

\subsection{The profiling radiometer RPG-HATPRO}

The RPG-HATPRO is a ground-based passive microwave radiometer, which operates in two frequency bands (Rose et al, 2005). It has seven channels on the upper wing of the $22 \mathrm{GHz}$ water vapor absorption line and seven channels at the $60 \mathrm{GHz}$ oxygen absorption complex: the line around $22 \mathrm{GHz}$ is used to retrieve humidity information; the second one allows retrieving temperature profiles. HATPRO measures brightness temperatures with a resolution of up to $1 \mathrm{~s}$. It has two operation modes: zenith pointing ( $\mathrm{Z}$ mode) and elevation scanning mode ( $S$ mode). In the $\mathrm{Z}$ mode the radiometer points vertically, while in the $\mathrm{S}$ mode it scans the atmosphere under elevation angles between about $4^{\circ}$ and $90^{\circ}$. By the assumption of horizontal homogeneity of the atmosphere, the received radiation for optically thick channels originates from lower atmospheric layers if the elevation angle is reduced. The scanning mode is used only for temperature retrieval, because in the water vapor band the optical thickness of the atmosphere is too low. In addition, the assumption for water vapor to be horizontally homogeneous is rarely valid.

Since the detected intensities have to be transferred to brightness temperatures, the radiometer needs to be well cal- ibrated. The absolute calibration consists in scanning blackbody targets whose radiometric temperature is considered to be equal to the physical one. In order to preserve the stability of the brightness temperature it is necessary to perform an absolute calibration with the liquid nitrogen cooled target; therefore, we have performed a liquid nitrogen calibration every 5 months. Automatic calibration includes the calibration of the receiver gain. The overall brightness temperature accuracy reported by the manufacturer for the RPGHATPRO, accounting for the receiver noise, receiver drifts and accuracy of calibrations, is $0.5 \mathrm{~K}$. A complete description of the calibration is given in Rose et al. (2005).

\subsection{Experimental setup}

The RPG-HATPRO radiometer is installed on the roof of the Institute for Meteorology and Geophysics (IMGI), University of Innsbruck, at an altitude of $612 \mathrm{~m}$ a.s.l. and has been operational since 1 September 2012. It is programmed to operate in both zenith $(\mathrm{Z})$ and scanning $(\mathrm{S})$ modes. Therefore it is oriented to scan along the Inn Valley axis towards the northeast $\left(60^{\circ} \mathrm{N}\right)$. The radiometer operates in both $\mathrm{Z}$ and $\mathrm{S}$ modes every $5 \mathrm{~min}$. The time repetition between the measurement modes (TP-BL repetition) and the integration times are shown in Table 4.

Austro Control, the Austrian Air Navigation Service, performs a radio sounding at Innsbruck airport every night at about 02:00 UTC. The launch site is located at a distance of about $3 \mathrm{~km}$ from the IMGI at an altitude of $577 \mathrm{~m}$ a.s.1. The comparison between current sounding and retrieved profiles is performed after a quality check of both HATPRO and radiosonde data and after deleting the days with rain flag switched on (cf. following subsection). Radiosonde measurements have an accuracy $\left(2 \sigma\right.$ confidence level) of $0.5^{\circ} \mathrm{C}$ for 
Table 4. Setup for the time repetition and time integration of our RPG-HATPRO. TP-BL repetition indicates the time between two successive scanning measurements. The measured brightness temperature is integrated over a certain time interval (level 1) and the retrieved profiles are then averaged (level 2).

\begin{tabular}{|c|c|}
\hline TP-BL repetition & $300 \mathrm{~s}$ \\
\hline $\begin{array}{l}\text { Level 1: time integration bright- } \\
\text { ness temperature }\end{array}$ & $\begin{array}{l}\mathrm{Z} \text { mode: } 1 \mathrm{~s} \\
\mathrm{~S} \text { mode: } 150 \mathrm{~s}\end{array}$ \\
\hline $\begin{array}{l}\text { Level 2: time integration re- } \\
\text { trieved profiles }\end{array}$ & $\begin{array}{l}\mathrm{Z} \text { mode: } 60 \mathrm{~s} \\
\mathrm{~S} \text { mode: } 150 \mathrm{~s}\end{array}$ \\
\hline
\end{tabular}

the temperature and $5 \%$ for the relative humidity (Vaisala, 2013).

As the radiosonde launches are performed each night at about 02:00 UTC, the HATPRO profile closest in time is taken for comparison. The radiosonde data are available at a temporal resolution of $2 \mathrm{~s}$, corresponding to a mean height of $8-10 \mathrm{~m}$. These data are interpolated to the HATPRO grid (Table 1) for further analysis. The different heights of the airport and the roof of IMGI $(+35 \mathrm{~m})$ are taken into account, as the temperature may change significantly over this height range.

\section{Data quality check}

For the radiosonde data the following checks (physical consistency) are performed:

- pressure within the $1-1050 \mathrm{hPa}$ range and decreasing with height;

- temperature within the $210-330 \mathrm{~K}$ range;

- surface pressure larger than $500 \mathrm{hPa}$.

For the brightness temperatures from the radiometer the following conditions are checked:

- rain flag (any amount of rain measured) not activated;

- brightness temperature in the $2.7-330 \mathrm{~K}$ range;

- visual inspection to exclude spikes, caused by radio frequency interferences or other disturbances (e.g., aircraft, birds).

\section{Results}

\subsection{Scanning vs. zenith}

In order to give a first impression of the performance we present a temperature profile comparison between radiosonde and microwave radiometer profiles using the original RPG-algorithm with both scanning and zenith modes

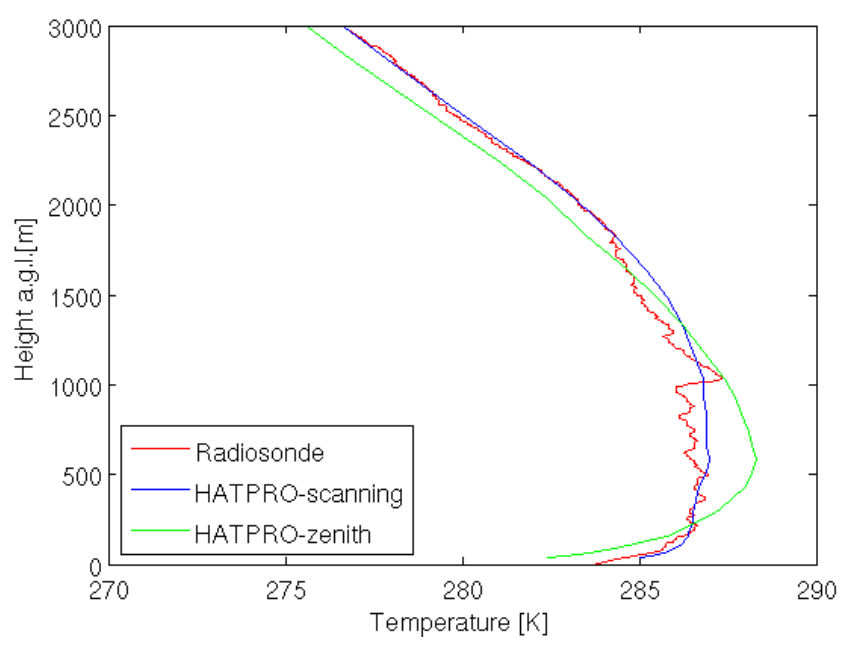

Figure 2. Temperature vertical profile for the lower part of the troposphere. The comparison is between (differently colored lines, see inlet) radiosonde and measurements of the radiometer by the RPGalgorithm with the scanning and zenith mode. Data from 9 September 2012 at 01:58 UTC (launch time of the radiosonde). The height is expressed in meters above ground.

(Fig. 2). This is a case with a deep near-surface temperature inversion throughout the first $1000 \mathrm{~m}$ above ground. The scanning mode gives a better shape of the temperature profile than the zenith mode. This is in agreement with the fact that the scanning mode yields more information about the lower atmospheric layers. For the upper atmosphere the accuracy of the temperature profiles with the two modes is generally similar (not shown), also in agreement with the findings by Crewell and Löhnert (2007).

\subsection{Evaluation of improved retrieval algorithms}

As explained in Sect. 2.3, the IMGI algorithm (cf. Table 3) is obtained using more robust statistics by using more and better resolved radiosonde data. Both IMGI and RPG algorithms are now applied to the full microwave radiometer data set (September 2012-May 2013). During this time, 84 radiosondes were launched and passed quality control. Figure 3 shows the mean RMSE computed between retrieved temperature profiles and radiosondes for this data set.

The profiles retrieved by the newly developed IMGI algorithm show an overall improvement at all levels with respect to the RPG algorithm. The errors of temperature profiles retrieved with the IMGI algorithm (Fig. 3) are considerably reduced compared to RPG algorithm in nearly all atmospheric layers. The largest improvement can be found in the upper troposphere, but also within the lowest $1000 \mathrm{~m}$ the errors are smaller, thus advantageous for studying the boundary layer structure.

More specifically, the RMSE for temperature with the IMGI algorithm is between 0.3 and $0.7 \mathrm{~K}$ below $500 \mathrm{~m}$ above ground; within $0.9 \mathrm{~K}$ in the layer between 500 and $1200 \mathrm{~m}$, 


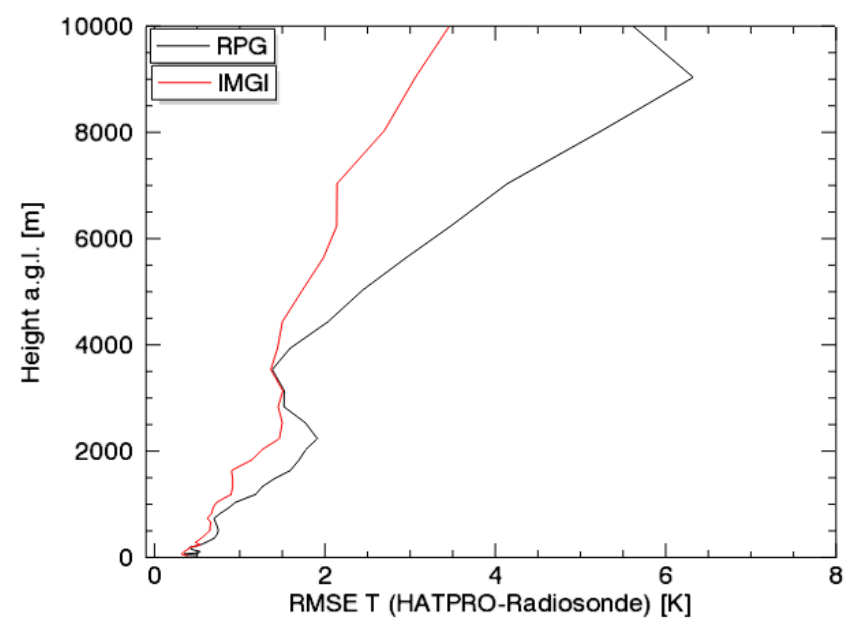

Figure 3. Comparison between RPG and IMGI algorithms for temperature profiles within the full troposphere. The RMSE is between temperature retrieved from the two algorithms and radiosonde in function of height (above ground). The 84 days refer to the time period from September 2012 to May 2013 and are all during the night. The radiosonde measurements are from the airport of Innsbruck and the profiler is located on the roof of IMGI.

it increases to $1.5 \mathrm{~K}$ at $4000 \mathrm{~m}$ and stays within $3.5 \mathrm{~K}$ at $10000 \mathrm{~m}$. Our results compare well and are even slightly better than those of previously published studies. One of the most recent and statistically most robust results of temperature profiles retrieved by ground-based radiometry is from Löhnert and Maier (2012). They used observations from the same type of radiometer at the aerological station of Payerne at $456 \mathrm{~m}$ a.s.l. (MeteoSwiss) and compared their profiles with collocated radiosonde data. They found an RMSE between retrieved profiles and radiosonde data to be between 0.4 and $0.8 \mathrm{~K}$ in the lowest $500 \mathrm{~m}$, within $1.2 \mathrm{~K}$ at $1200 \mathrm{~m}$ and around $1.7 \mathrm{~K}$ at $4000 \mathrm{~m}$ above ground.

This improved performance is very encouraging given the fact that Löhnert and Maier (2012) used a significantly larger radiosonde data set (of about 12000 high-resolution radiosondes during both daytime and nighttime) for their retrieval, providing a more robust diurnal cycle but also more robust statistics in general than we had at our disposal. Moreover, and probably more importantly, the obtained performance being equal (or even slightly better than) to that from less complex terrain demonstrates that microwave radiation originating from nearby slopes (or terrain in general) is not likely to deteriorate the quality of vertical profile information. Hence, the use of HATPRO for the continuous monitoring of the boundary layer (and beyond) in a mountain valley is possible and promising - at least if the scanning mode is performed in along-valley direction as in the present case.

The IMGI algorithm also outperforms RPG algorithm for humidity profiles (Fig. 4). The improvement is observed throughout the full troposphere and it is especially pronounced in the lower atmospheric layers. In particu-

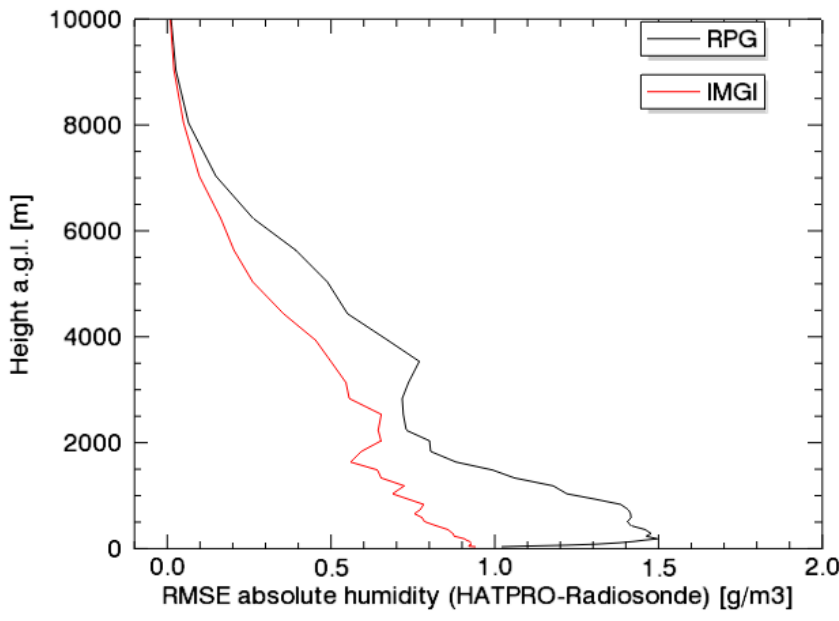

Figure 4. Same as Fig. 3, but for absolute humidity.

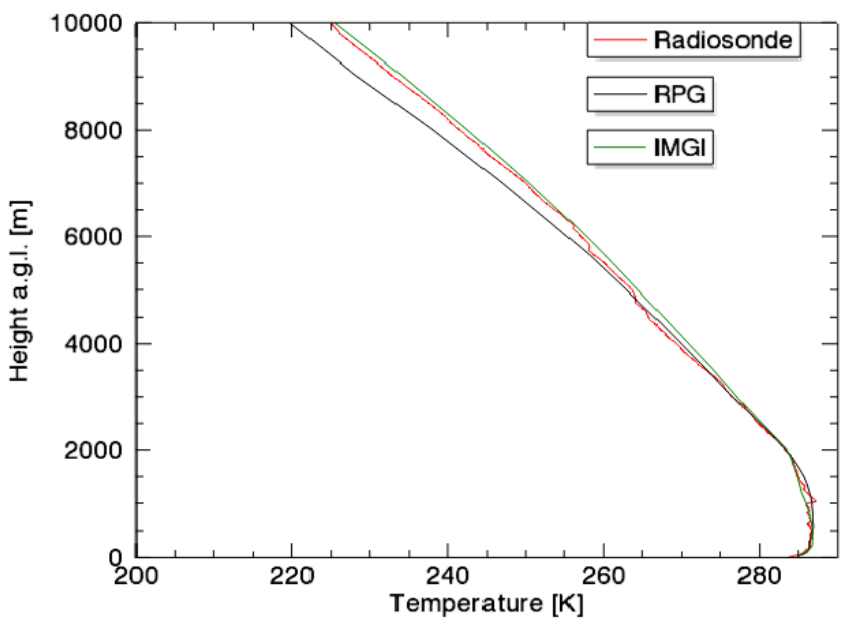

Figure 5. Temperature profile within the full troposphere. The comparison is between (differently colored lines, see inlet) radiosonde, RPG algorithm, IMGI algorithm. They refer to 9 September 2012 at 01:58 UTC (launch time of the radiosonde). The height is expressed in meters above ground.

lar, the RMSE of absolute humidity with the IMGI algorithm is between 0.9 and $0.8 \mathrm{~g} \mathrm{~m}^{-3}$ below $500 \mathrm{~m}$; within $0.7 \mathrm{~g} \mathrm{~m}^{-3}$ at $1200 \mathrm{~m}$, it decreases to $0.4 \mathrm{~g} \mathrm{~m}^{-3}$ at $4000 \mathrm{~m}$ and to $0.01 \mathrm{~g} \mathrm{~m}^{-3}$ at the top of the troposphere. Löhnert et al. (2009) found an RMSE of absolute humidity around $0.8 \mathrm{~g} \mathrm{~m}^{-3}$ in the lowest $500 \mathrm{~m}$, within $0.7 \mathrm{~g} \mathrm{~m}^{-3}$ at $1200 \mathrm{~m}$ and within $0.4 \mathrm{~g} \mathrm{~m}^{-3}$ at $4000 \mathrm{~m}$ above ground retrieving the absolute humidity profiles from brightness temperatures that were measured by a HATPRO in Payerne and comparing them with radiosonde data. Taking into account that the results by Löhnert et al. (2009) were obtained using a more sophisticated optimum estimation technique and were based on synthetic data, our results for humidity profiles can be considered very satisfying. 


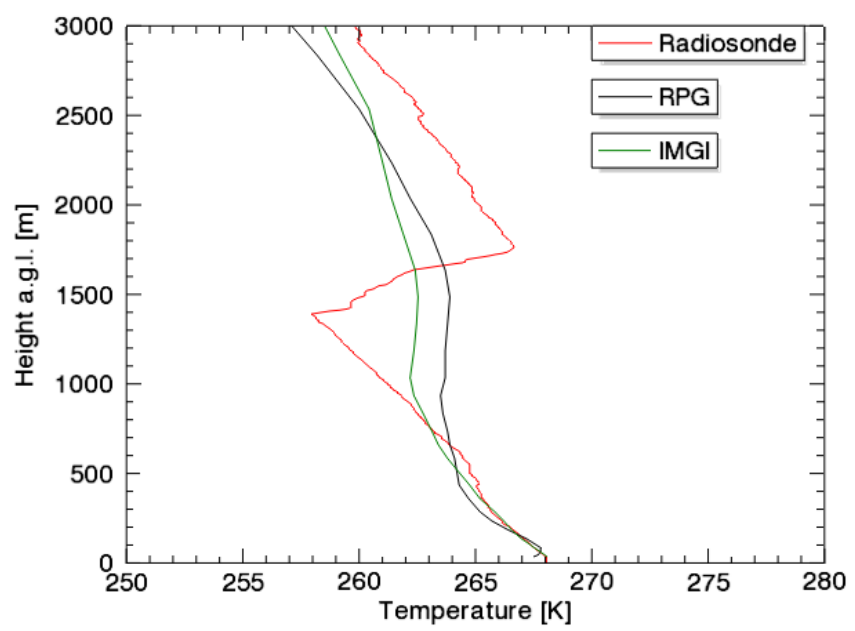

Figure 6. Temperature vertical profile for the lower part of the troposphere. The comparison is between (differently colored lines, see inlet) radiosonde, RPG algorithm, IMGI algorithm. They refer to 14 September 2013 at 03:18 UTC (launch time of the radiosonde). The height is expressed in meters above ground.

With the knowledge of improved statistical performance, we now evaluate our new IMGI retrieval for a couple of inversion cases. Figure 5 shows the same case of a deep nighttime inversion as in Fig. 2, but for the retrieved temperature profiles with the RPG and IMGI algorithms. Both algorithms capture the inversion, but the IMGI reproduces the temperature gradient slightly better; in the middle to high troposphere the better performance of the IMGI algorithm is evident. Figure 6 shows a night with a very strong elevated temperature inversion around $1500 \mathrm{~m}$ above ground. Both the RPG and the IMGI algorithms reproduce it only roughly. This type of inversion case is nearly impossible to retrieve by radiometers when only ground-based measurements are available. Similar underperformance has already been shown by Löhnert and Maier (2012) using the same instrument and by Knupp et al. (2009), operating a similar profiling radiometer (manufactured by Radiometrics Corporation). This is likely due to the height of the inversion, which is higher than the atmospheric layers that benefit from the scanning mode (see, e.g., Crewell and Löhnert, 2007); hence the radiometer information is not sufficient to resolve it. Additionally, a much larger number of cases in the training data set showing an elevated inversion of this type (note that in the current training data set this number is quite limited) might slightly improve the statistics but clearly will not overcome this problem of poor resolution aloft.

An example of a retrieved nighttime humidity profiles is shown in Fig. 7. Only the mean shape of the actual humidity profile is reproduced by both retrievals, but for the present example the IMGI algorithm yields a slightly better overall accuracy. This is related to the transparency of the atmosphere in the K-band and in accordance with the findings by Lilje-

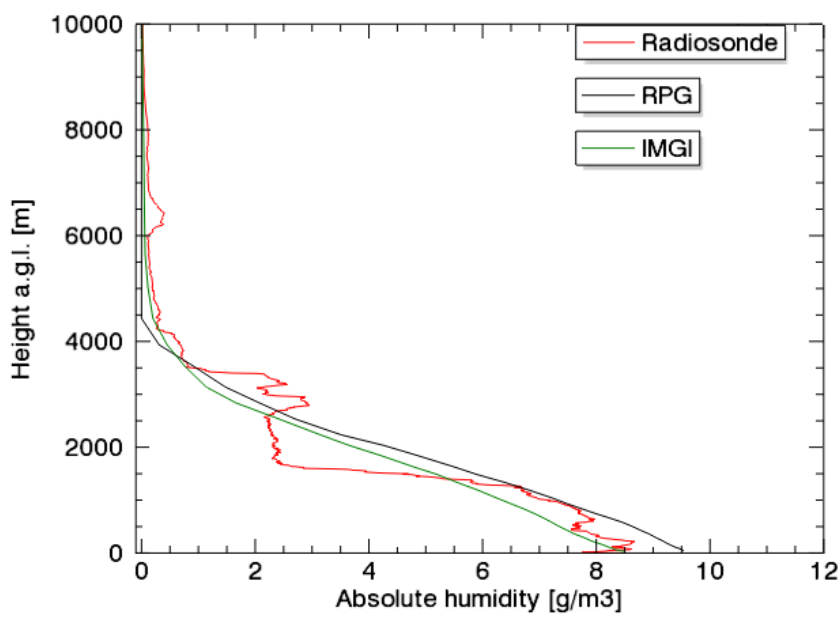

Figure 7. Humidity vertical profile within the full troposphere. The comparison is between (differently colored lines, see inlet) radiosonde, RPG algorithm, IMGI algorithm. They refer to 3 September 2012 at 02:02 UTC (launch time of the radiosonde). The height is expressed in meters above ground.

gren et al. (2001) and Ware et al. (2003), using similar passive microwave radiometers. The lower information on the vertical humidity distribution is caused by the lower information content for water vapor than for temperature observations; for humidity profiles there are 1-2 independent degrees of freedom, whereas for temperature profiles the scanning measurement contains 4 independent pieces of information (Löhnert et al., 2009).

\subsection{Impact of targeted training data sets}

Further improvement to the retrieved profiles was expected by performing separate retrieval development for targeted data sets, for example seasonal or time-of-day subsets (see Sect. 2.3.1). Unfortunately, the number of available daytime radiosonde launches is very small (only 134); hence the statistical analysis is biased towards the nighttime cases. In order to develop robust statistics for daytime cases, 10 different independent random choices were performed for this subset. The results are displayed in Fig. 8. The nighttime retrieval (N) roughly follows the behavior of the general IMGI retrieval (All), according to the fact that the number of nighttime radiosonde measurements is predominant. Daytime (D), winter $(\mathrm{W})$ and winter nighttime $(\mathrm{WN})$ retrievals have worse RMSE than IMGI retrieval (difference larger than $0.1 \mathrm{~K}$ ). On the other hand, retrieval algorithms based on the summer (S) or summer nighttime (SN) appear to give better results (difference in RMSE larger than $0.1 \mathrm{~K}$ ). The results for cold and warm months' retrievals are equivalent to those of winter and summer respectively and are therefore not shown in Fig. 8. The results for the daytime retrieval are not very conclusive due to the small number of samples. 


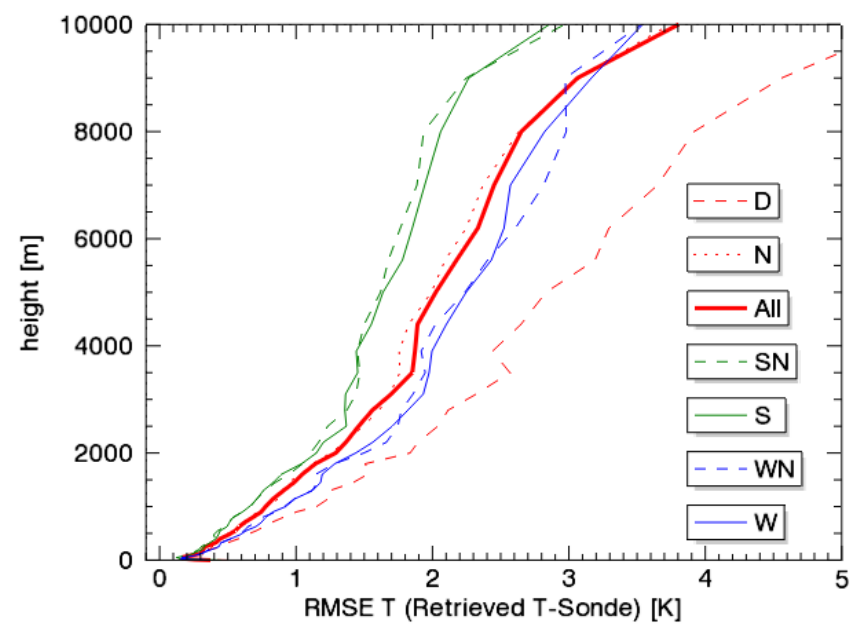

Figure 8. Effect of a specialized training considering time of year or time of day. RMSE between IMGI retrieved temperature and radiosonde in function of height (above the radiometer) for the full troposphere. D: daytime radiosonde (134); N: nighttime (3023); All: overall set of radiosonde (3157); SN: summer nighttime (984); S: summer (1028); WN: winter nighttime (990); W: winter (1026).

The statistics for summer and winter retrievals are quite robust (more than 1000 profiles). However, in winter (or in the cold months) it seems to be more difficult to retrieve the temperature profile. The differences between summer and winter can be partly explained by more frequent elevated and multiple inversions in winter. Moreover, in mid-tropospheric levels, the natural atmospheric variability is lower in summer, resulting in a smaller climatic RMSE.

Concerning the diurnal cycle the daytime boundary layer is often characterized by an elevated inversion (entrainment layer during thermally dominated situations); on the other hand for dynamically dominated situations often no inversions are present. Therefore, apparently "daytime" is a criterion that is not sufficiently unique to benefit from a specialized retrieval.

The results show that, with careful selection of climatic subsets, the resulting profile algorithms can yield better results. However, for transition periods between different seasons or day/night a combined algorithm would be required in order to continuously monitor atmospheric profiles.

Apparently, summer profiles are overall more similar among themselves than the winter profiles. Likely due to the abundance of nighttime profiles in the present data set, the summer profiles have no or only surface inversions, whereas the group of winter profiles possesses many cases of elevated or even multiple inversions. The fact that there are largely improved results for the $\mathrm{S}$ and $\mathrm{SN}$ specialized retrievals, in principle, suggests that a better selection of the training profiles can yield better results. However, only if physically meaningful specializations are chosen - and if these groups have

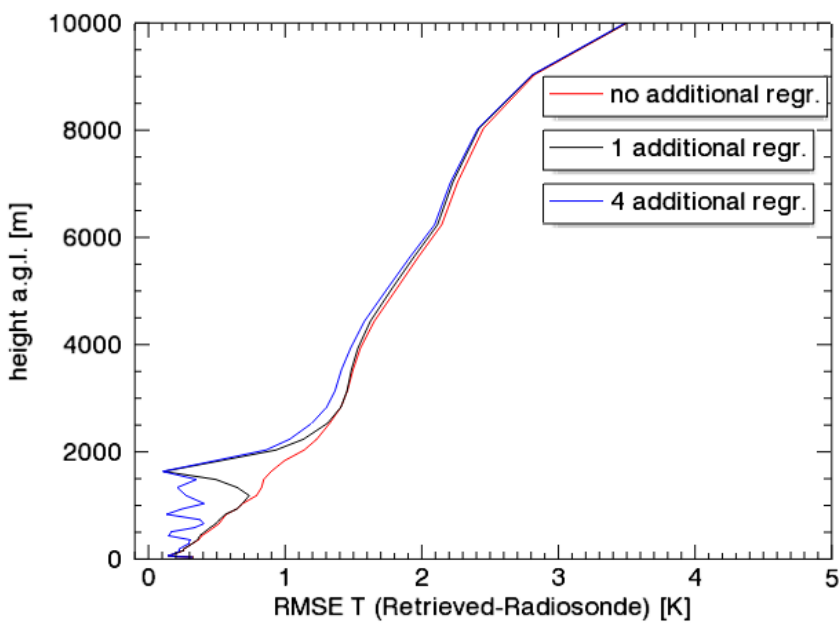

Figure 9. Comparison between a retrieval based only on the brightness temperatures (called "no additional regr."), a retrieval with the temperature at $1600 \mathrm{~m}$ (called "1 additional regr.") and a retrieval with the temperature at several heights $(400,800,1200,1600 \mathrm{~m})$ as further regressors (called " 4 additional regr."). The RMSE is between retrieved temperature and radiosonde in function of height (above ground).

a sufficiently large database - the targeted specialized retrievals can be beneficial.

\subsection{Additional regressors}

Another potential method for improving the accuracy of the retrieval is including additional regressors into the algorithm development (Eq. 2), like the pressure or temperature at the height of the radiometer. These data have been shown to improve the temperature and humidity retrievals in the lower atmosphere (Del Frate and Schiavon, 1998). Alternatively, in mountainous regions, such as the Inn Valley, it is possible to include pressure and temperature measurements from meteorological stations on the surrounding mountains. One such example is a weather station at Patscherkofel in the vicinity of Innsbruck, at about $1600 \mathrm{~m}$ above the valley floor, and at only $8 \mathrm{~km}$ horizontal distance from the microwave radiometer location. More generally, in such a terrain the temperature at specific altitudes can be routinely available.

As a proof of concept, we test two new retrievals. The first one uses the temperature at $1600 \mathrm{~m}$ above the valley floor as the additional information and the second retrieval uses "a slope profile", i.e., temperatures at $400,800,1200,1600 \mathrm{~m}$ (above the valley floor) as additional regressors.

Note that these temperatures (regressors) are here obtained from the soundings and not from actual surface measurements at these altitudes. Figure 9 shows the RMSE comparison between the retrievals with additional regressors and the IMGI retrieval without them. It is apparent that the accuracy of temperatures at the heights close to those of additional regressors is significantly improved (the RMSE is 


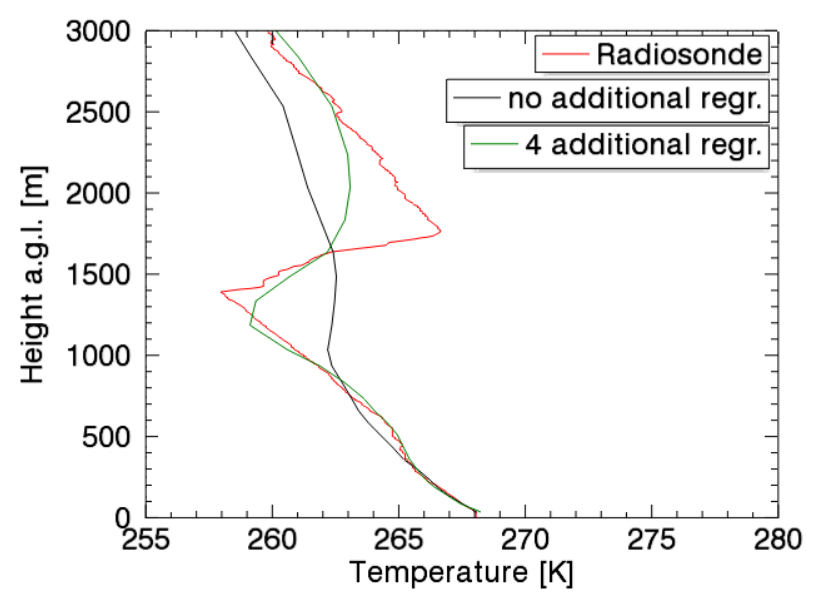

Figure 10. The effect of additional regressors for a real case that refers to 14 February 2013 at 03:18 UTC (launch time of the radiosonde). The comparison is between the radiosonde profile, a retrieval based only on the brightness temperatures (called "no additional regr.", corresponding to the IMGI algorithm) and a retrieval with the temperature at several heights $(400,800,1200,1600 \mathrm{~m})$ as further regressors (called " 4 additional regr."). The height is above ground.

about $0.1 \mathrm{~K})$. The beneficial effect of these additional regressors is most pronounced within a zone of several hundred meters around the height of the additional temperatures themselves. Still, the overall RMSE is improved also up to the mid-troposphere, especially for four additional regressors constraining the retrieved profile. Therefore, the disadvantage of operating the radiometer in mountainous terrain, with frequent higher-level inversions that are challenging for standard radiometer retrievals, can potentially be turned into a benefit; the resolution of the radiometer profile in the lower troposphere might be improved in a mountainous region where additional surface measurements from different altitudes (e.g., along a nearby slope or from mountain peaks) are available.

The potential of such an approach becomes clear when considering an example with a pronounced elevated inversion, which both the RPG and IMGI retrievals were unable to resolve properly. Figure 10 shows the same case as Fig. 6 but for the IMGI retrieval and the new retrieval with four additional regressors. The retrieval algorithm with the information from elevated levels included provides a significantly better representation of the inversion than the nonspecialized (IMGI) algorithm, both in terms of inversion height and temperature gradient. Naturally, the improvement is largest at levels where the additional information comes from.

The additional regressors used in the present "proof of concept" are the actual temperatures at various heights in the valley atmosphere. The measurements one can possibly have at disposition, however, even in complex mountainous ter- rain with steep slopes and nearby peaks are surface based observations, e.g. along a slope or on mountain tops of different heights. Some decades ago a number of comparisons between slope profiles and free air temperature profiles (even for the Inn Valley) were performed (e.g., Brehm and Freytag 1982; Dreiseitl, 1988). Clearly, a slope observation is influenced by nearby terrain and thus a slope profile will not, in general, be equal to that in the valley boundary layer. More recent simulations using meso-scale numerical models and idealized topography (e.g., Wagner et al., 2014) show that isentropes are not horizontal in a valley atmosphere (at least under convective conditions as in their case) due to the local baroclinicity of the flow. Thus, the slope profile will not detect an elevated inversion at the correct height - but likely at a consistently different height. Numerical simulations using idealized terrain might suffer from over-idealization, i.e., in the present case the (assumed) symmetry with respect to solar insolation on the slopes. Matzinger et al. (2003) have shown that net radiation (essentially providing the energy to heat the slopes) can be up to a factor of 3 different at different positions in a north-south-oriented valley in mid-latitudes under convective conditions and the surface heat fluxes vary correspondingly (Rotach et al., 2008). Turbulent mixing and the resulting cross-valley, thermally driven circulation, however, will have a tendency to alleviate these contrasts. Laiti et al. (2013) have used spatial interpolation techniques to obtain cross-valley temperature distributions based on airborne observations (for a small valley in the Italian Alps). Clearly, the isentropes are not horizontal for many of their flights and cross sections but they appear to be "sufficiently uniform" to at least make it plausible that a slope-based profile might add some additional information to improve the retrieval if included in the algorithm.

In this sense, it can be hypothesized that a slope-based profile will exhibit the gross features of elevated inversions (such as in Fig. 10) and is likely to considerably improve the overall performance of the retrieval. This approach is currently being tested on the basis of actual slope profiles in the vicinity of our profiler - and results will be detailed in a later contribution. It will also be interesting to find out up to which horizontal distance from a profiler location a mountaintop measurement site would have a beneficial impact on the retrieval algorithm.

\subsection{Boundary layer dynamics}

In order to demonstrate the potential of a microwave radiometer for describing the boundary layer dynamics in an alpine valley, the daily cycle of temperature for the night where we found a strong elevated inversion around $1500 \mathrm{~m}$ (cf. Fig. 10) is displayed in Fig. 11. The inversion (Fig. 10) starts to grow around 03:00 UTC and develops throughout the morning hours and results in an almost isothermal (i.e., stable) valley atmosphere before noon. The surface heating in the afternoon produces a shallow layer of nearly neu- 


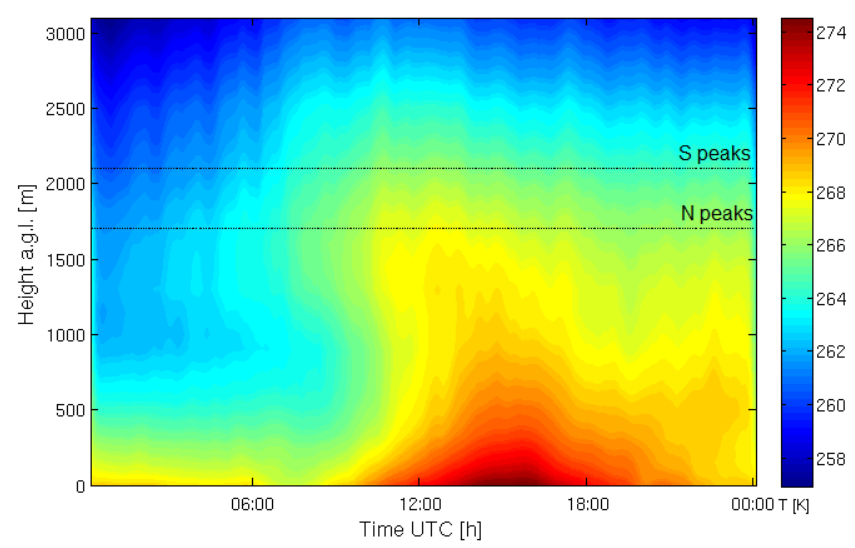

Figure 11. Daily temperature evolution for 14 February 2013 obtained from IMGI retrieval (with no additional regressors) in the lower part of atmosphere. The time is expressed in UTC and the height is expressed in meters above ground level (a.g.1.). The dotted horizontal lines correspond to the approximate peak heights towards the north $(\mathrm{N}$, about $1700 \mathrm{~m})$ and south of the valley $(\mathrm{S}$, about $2100 \mathrm{~m})$.

tral stratification near the ground while the remainder of the valley atmosphere remains stable $\left(\partial T / \partial z \approx-0.003 \mathrm{~km}^{-1}\right)$ throughout the afternoon. Above the crest height the somewhat weaker stratification of the large-scale flow persists throughout the day. Apparently, the flow interaction between the free troposphere and the valley atmosphere is largely constrained by the surrounding topography under the wintertime conditions of Fig. 11. For more convective conditions, numerical simulations using idealized terrain (Wagner et al., 2014) suggest that - depending on the dimensions of the valley - the stability characteristics of the valley atmosphere can be confined to below crest height or extend up to much higher altitudes. The example of Fig. 11 demonstrates both the potential of a microwave temperature profiler to resolve the temporal development much better than a sequence of soundings could - and this is of course not specific for mountainous terrain - as well as the necessity to optimally resolve elevated inversions in this type of environment.

\section{Conclusions and outlook}

Within the $i$-Box Project a RPG-HATPRO microwave radiometer was operated for one of the first times during a prolonged period in an alpine valley, i.e., in truly complex terrain. Although the atmospheric boundary layer is not homogeneous in this terrain, the radiometer allows obtaining continuous temperature and humidity profiles with acceptable accuracy and shows no deterioration of the retrieved profile due to the influence of microwave radiation from the nearby terrain. These profiles can be used for studying boundary layer processes in complex mountainous terrain. Observations show that by using the elevation scanning mode more accurate temperature profiles can be retrieved in the lowest $1200 \mathrm{~m}$ above ground, as already reported by Crewell and Löhnert (2007). Furthermore, we could show that we were able to improve the retrieval algorithm even further.

First, temperature and humidity profiles retrieved by the algorithm provided by the manufacturer (RPG algorithm) and an improved retrieval (IMGI algorithm), based on more robust statistics, have been compared for simultaneous nighttime radiosondes. The more robust statistics consists of a larger radiosonde data set, using their full vertical resolution, finding the best noise level and determining the best regression type (linear or quadratic). Considering 84 nights between September 2012 and May 2013 for which the radiometer and radiosonde data are available, the IMGI algorithm gives improved results both for temperature and absolute humidity. It can be concluded that the set of training data, i.e., the local climatology, is essential for obtaining accurate profiles. Specifically, the accuracy of the retrieved temperature profile with the IMGI retrieval is comparable to those obtained from other radiometers operated in less complex terrain (Löhnert and Maier, 2012; Löhnert et al., 2007) and at certain height ranges even better. It is thus shown that with the availability of a local, long-term, high-resolution training data set a microwave radiometer can be operated even in truly complex terrain.

On the other hand, neither the RPG nor the improved retrieval algorithms are able to accurately capture elevated temperature inversions at the level of the valley crest (typically above $1200 \mathrm{~m}$ in the present environment). It is known that these cases pose significant challenges to radiometry (Crewell and Löhnert, 2007). The most promising approach in complex mountainous terrain would be to include additional regressors from mountain stations into the retrieval. Our results show that the accuracy of the retrieved temperatures improves considerably at heights around those of additional regressors (despite the fact that the information on the height of those regressors is not included in the retrieval). Also and perhaps more importantly, the retrieval algorithm with these additional regressors is able to resolve an elevated inversion to an unprecedented degree.

Where information from higher levels (e.g., mountain stations) is not available, a better accuracy for temperature profiles can be obtained by training based on subsets of data with similar characteristics, thus allowing for a specialized retrieval for specific conditions. Our results for different seasons and nighttime-daytime show some improvements. However, the fact that there are better results for some classification subsets and worse for others suggests that targeted specialized retrievals based on classification of profiles according to their similar physical characteristics could give improved results. For this purpose, a more in-depth study of the typical profiles in the environment where the profiler is used would be required, as well as an appropriate method for classifying them. These however are out of the scope of this paper. Other types of training, such as artificial neural net- 
work, might be tested as well for this purpose. A powerful method of classification of the profiles could be in terms of their atmospheric stability.

Finally, when considering humidity profiles, details within the boundary layer cannot be resolved precisely, due to the limited information content of HATPRO observations. In order to obtain a better accuracy of retrieved humidity, it is likely necessary to use additional frequencies (e.g., $183 \mathrm{GHz}$ ) where the water vapor absorption is higher or to operate the radiometer in synergy with other remote-sensing instruments (e.g., Raman lidar). Also, humidity observations on slopes or mountaintops could act as additional regressors like we presented for temperature profiles.

Acknowledgements. The authors thank Austro Control of Innsbruck for providing with the radiosonde data. G. Massaro acknowledges the Erasmus Programme funding and E. Martines (National Research Council, Italy), who allowed him to take part in this research at the University of Innsbruck as part of his degree at the University of Padua. The authors also thank R. Stauffer and J. Messner (IMGI) for the fruitful discussions in statistics.

Edited by: S. J. Munchak

\section{References}

Bougeault, P., Binder, P., Buzzi, A., Dirks, R., Kuettner, J., Houze, R., Smith, R.B., Steinacker, R., and Volkert, H.: The MAP Special Observing Period, Bull. Amer. Meteor. Soc. 82, 433-462, 2001.

Brehm, M. and Freytag, C.: Erosion of the Night-Time Thermal Circulation in an Alpine Valley Arch, Met. Geoph. Biokl., Set. B, 31, 331-352, 1982.

Crewell, S. and Löhnert, U.: Accuracy of boundary layer temperature profiles retrieved with multifrequency multiangle microwave radiometry, IEEE T. Geosci. and Remote, 45, 2195-2201, 2007.

Del Frate, F. and Schiavon, G.: A combined natural orthogonal functions/neural network technique for the radiometric estimation of atmospheric profiles, Radio Sci., 2, 405-410, 1998.

Divakarla, M. G., Barnet, C. D., Goldberg, M. D., McMillin, L. M., Maddy, E., Wolf, W., Zhou, L., and Liu, X.: Validation of atmospheric infrared sounder temperature and water vapor retrievals with matched radiosonde measurements and forecasts, J. Geoph. Res., 111, D09S15, doi:10.1029/2005JD006116, 2006.

Dreiseitl, E.: Slope and Free Air Temperature in the Inn Valley, Meteorol. Atmos. Phys., 39, 25-41, 1988.

Güldner, J.: A model-based approach to adjust microwave observations for operational applications: results of a campaign at $\mathrm{Mu}-$ nich Airport in winter 2011/2012, Atmos. Meas. Tech., 6, 28792891, doi:10.5194/amt-6-2879-2013, 2013.

Harnisch, F., Gohm, A., Fix, A., Schnitzhofer, R., Hansel, A., and Neininger, B.: Spatial distribution of aerosols in the Inn Valley atmosphere during wintertime, Meteor. Atmos. Phys., 103, 223235, 2009.

Janssen, M. A: Atmospheric Remote Sensing by Microwave Radiometry, Wiley, New York, 1993.
Karstens, U., Simmer, C., and Ruprecht, E.: Remote sensing of cloud liquid water, Meteorol. Atmos. Phys., 54, 157-171, 1994.

Knupp, K. R. , Ware, R. , Cimini, D. , Vandenberghe, F. , Vivekanandan, J., Westwater, E. , Coleman, T., and Phillips, D.: Groundbased passive microwave profiling during dynamic weather conditions, J. Atmos. Oceanic Technol., 26, 1057-1073, 2009.

Laiti, L., Zardi, D., de Franceschi, M., and Rampanelli, G.: Atmospheric boundary layer structures associated with the Ora del Garda wind in the Alps as revealed from airborne and surface measurements, Atmos. Res., 132/133, 473-489, 2013.

Liljegren, J., Lesht, B., Kato, S., and Clothiaux, E.: Initial evaluation of profiles of temperature, water vapour and cloud liquid water from a new microwave profiling radiometer, 11th American Meteorological Society Symposium on Meteorological Observations and Instruments, Albuquerque, New Mexico, 14-19 January 2001, 2001.

Löhnert, U. and Crewell, S.: Accuracy of cloud liquid water path from ground-based microwave radiometry - Part I: dependency on cloud model statistics, Radio Sci., 38, 8041, doi:10.1029/2002RS002654, 2003.

Löhnert, U., Turner, D. D., and Crewell, S.: Ground-based temperature and humidity profiles using spectral infrared and microwave observations - Part I: simulated retrieval performance in clearsky conditions, J. Appl. Meteor. Climatol., 48, 1017-1032, 2009.

Löhnert, U. and Maier, O.: Operational profiling of temperature using ground-based microwave radiometry at Payerne: prospects and challenges, Atmos. Meas. Tech., 5, 1121-1134, doi:10.5194/amt-5-1121-2012, 2012.

Matzinger, N., Andreatta, M., van Gorsel, E., Vogt, R., Ohmura, A., and Rotach, M. W.: Surface radiation budget in an Alpine valley, Q. J. R. Meteorol. Soc., 129, 877-895, 2003.

Rose, T., Crewell, S., and Löhnert, U.: A network suitable microwave radiometer for operational monitoring of the cloudy atmosphere, Atmos. Res., 75, 183-200, 2005.

Rosenkranz, P.: Water vapour continuum absorption: a comparison of measurements and models, Radio Sci., 33, 919-928, 1998.

Rotach, M. W. and Zardi, D.: On the boundary-layer structure over highly complex terrain: key findings from MAP, Q. J. R. Meteorol. Soc., 133, 937-948, 2007.

Rotach, M. W., Andretta, M., Calanca, P., Weigel, A. P., and Weiss, A.: Turbulence characteristics and exchange mechanisms in highly complex terrain, Acta Geophys., 56, 194-219, 2008.

Rotach, M. W., Stiperski, I., Gohm, A., Sfyri, E., Goger, B., Vergeiner, J., and Fuhrer, O.: The Innsbruck-Box ( $i$-Box) - an observational and modeling framework for the investigation of exchange processes over complex topography, Bull. Amer. Meteorolo. Soc., submitted, 2015.

Sanchez, J. L., Posada, R., Garcia-Ortega, E., Lopez, L., and Marcos, J. L.: A method to improve the accuracy of continuous measuring of vertical profiles of temperature and water vapor density by means of a ground-based microwave radiometer, Atmos. Res., 122, 43-54, 2013.

Steppeler, J., Doms, G. , Schättler, U., Bitzer, H., Gassmann, A., Damrath, U., and Gregoric, G.: Meso-gamma scale forecast using the non-hydrostatic model LM, Meteor. Atmos. Phys., 82, 75-96, 2003.

Stogryn, A. P., Bull, H. T., Rubayi, K., and Iravanchy, S.: The microwave dielectric properties of sea and fresh water, Tech. rep., GenCorp Aerojet, Azusa, California, USA, 1995. 
Vaisala: Vaisala Radiosonde RS92-SGP data sheet, Vaisala Oyj, Vantaa, Finland, 2013

Wagner, J. S., Gohm, A., and Rotach, M. W.: The impact of valley geometry on daytime thermally driven flows and vertical transport processes, Qart. J. Roy. Meteorol. Soc., 1477-870X, doi:10.1002/qj.2481, 2014.

Ware, R., Carpenter, R., Güldner, J., Liljegren, J., Nehrkorn, T., Solheim, F., and Vandenberghe, F.: A multichannel radiometric profiler of temperature, humidity and cloud liquid, Radio Sci., 38, 8079-8092, 2003.
Weigel, A. P. and Rotach, M. W.: Flow structure and turbulence characteristics of the daytime atmosphere in a steep and narrow Alpine valley, Quart. J. Roy. Meteorol. Soc., 130, 2605-2627, 2004.

Wolf, T., Isau, I., and Reuder, J.: Analysis of the vertical structure in the Bergen valley, Norway, and its connection to pollution episodes, J. Geophys. Res., 119, 10-645, 2014.

Zardi, D. and Whiteman, C. D.: Diurnal Mountain Wind Systems, Chapter 2, in: Mountain Weather Research and Forecasting, edited by: Chow, F. K., DeWekker, S. F. J., and Snyder, B., Springer, Berlin, 35-119, 2012.

Zhou, D. K., Smith, W. L., Liu, X., Larar, A. M., Mango, S. A., and Huang, H. L.: Physically retrieving cloud and thermodynamic parameters from ultraspectral IR measurements, J. Atmos. Sci., 64, 969-982, 2007. 